\title{
THE EFFECT ON NEONATAL RAT OFFSPRING BORN TO DAMS EXPOSED TO METHADONE OR BUPRENORPHINE DURING PREGNANCY
}

\author{
Mette Kongstorp ${ }^{1,2}$, Inger Lise Bogen ${ }^{1,2}$, Tom Stiris ${ }^{2,3}$, Jannike Mørch Andersen ${ }^{1,4}$
}

1) Department of Forensic Medicine, Oslo University Hospital, Oslo, Norway, 2) Faculty of Medicine, University of Oslo, Oslo, Norway, 3) Department of Neonatal Intensive Care, Oslo University Hospital, Oslo, Norway, 4) School of Pharmacy, University of Oslo, Oslo, Norway

\section{BACKGROUND}

Opioid maintenance treatment (OMT) with methadone or buprenorphine is the recommended therapy for heroin-dependent pregnant women. Compared to heroin use in pregnancy, OMT is associated with less illicit drug intake and better prenatal care [1]. However, there is a growing concern about possible negative outcomes of this treatment on the child. Such outcomes can be difficult to reveal in humans because of numerous cofounding factors. Experimental animal studies can provide useful information, but the human relevance of earlier animal studies has been questioned due to the use of high doses, intermittent administrations, rapid metabolism and missing data on blood opioid concentrations. In the present study we evaluated the human relevance of an exposure protocol aiming to maintain stable and clinical relevant blood concentrations of methadone or buprenorphine in pregnant rats, using osmotic minipumps implanted prior to mating. Opioid concentrations of administrated opioid were measured in blood during pregnancy, and in blood and brain from newborn pups. The offspring were also evaluated for neonatal outcomes related to growth and abstinences.

\section{METHODS}

Female rats were implanted with a 28-days osmotic minipump (Alzet, Cupertino, $\mathrm{CA})$, delivering methadone $(10 \mathrm{mg} / \mathrm{kg} / \mathrm{day})$, buprenorphine $(1 \mathrm{mg} / \mathrm{kg} /$ day $)$ or sterile water, 5 days before mating. Opioid concentrations were analyzed in blood from pregnant rats, and in blood and brain from newborn pups by use of an established LC-MS/MS method. Neonatal outcomes were investigated on postnatal day (PND) 1. Symptoms of neonatal abstinences were examined by ultrasonic vocalization (USV) [2] on PND 1, 3, 7 and 14, and by analysis of blood corticosterone levels [3] on PND 1, 7 and 14.

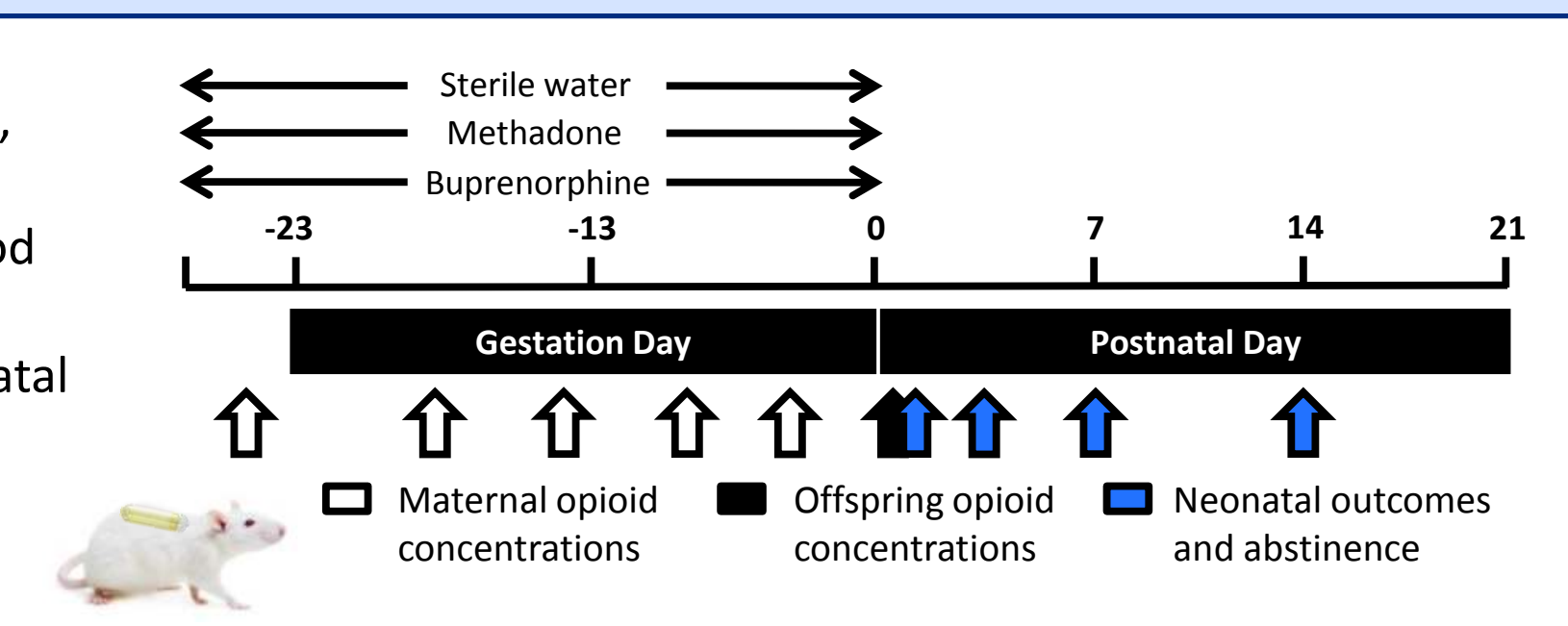

RESULTS

a)

\section{Maternal opioid concentrations}

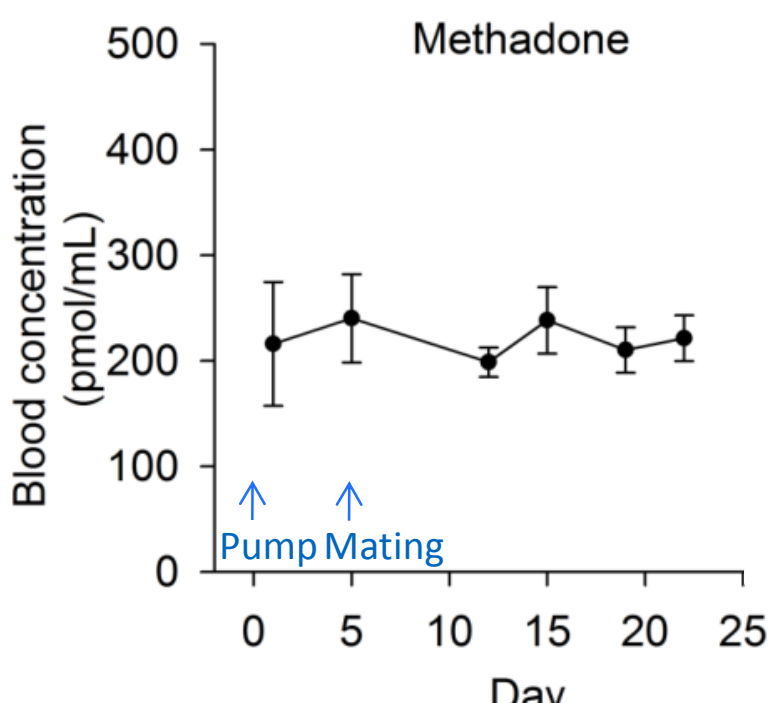

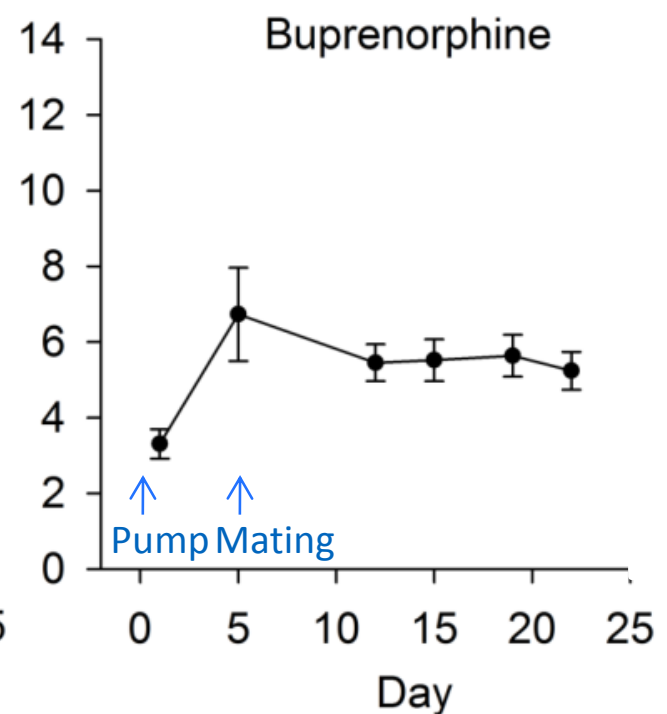

b)

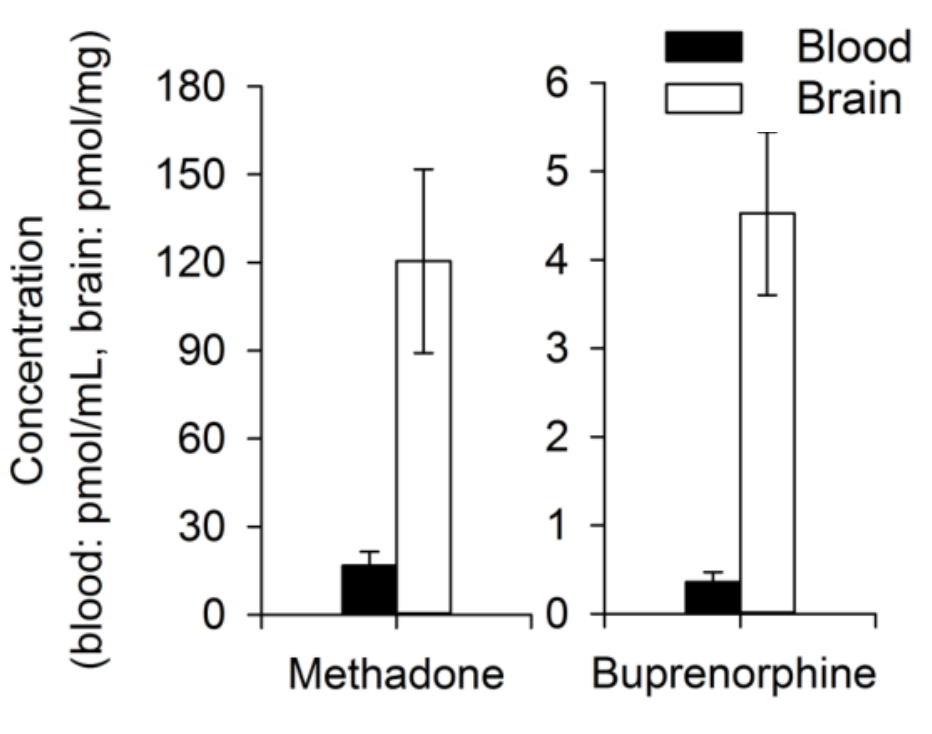

Figure 1. a) Maternal blood concentrations of methadone and buprenorphine prior to and during pregnancy. b) Blood and brain concentrations of methadone and buprenorphine in offspring on PND 1. Results are shown as mean \pm SEM, $n=8-10$.
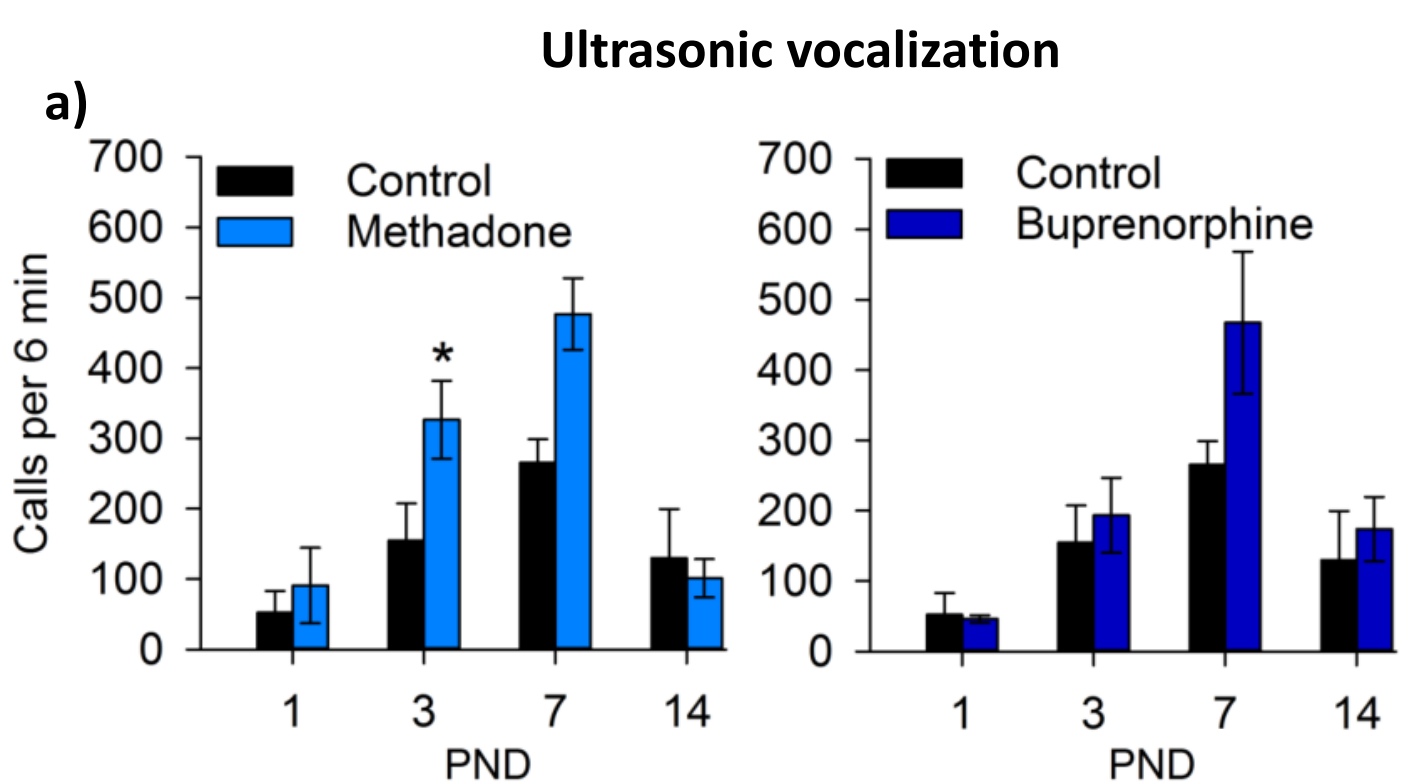

b)

\section{Corticosterone concentrations}

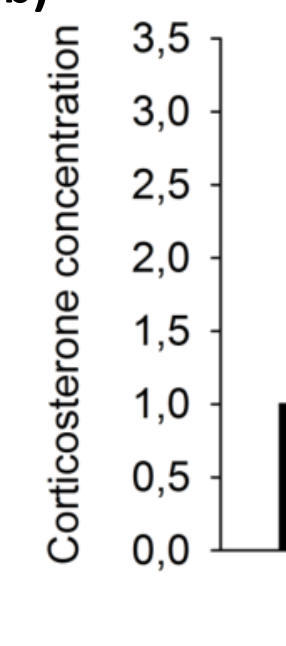

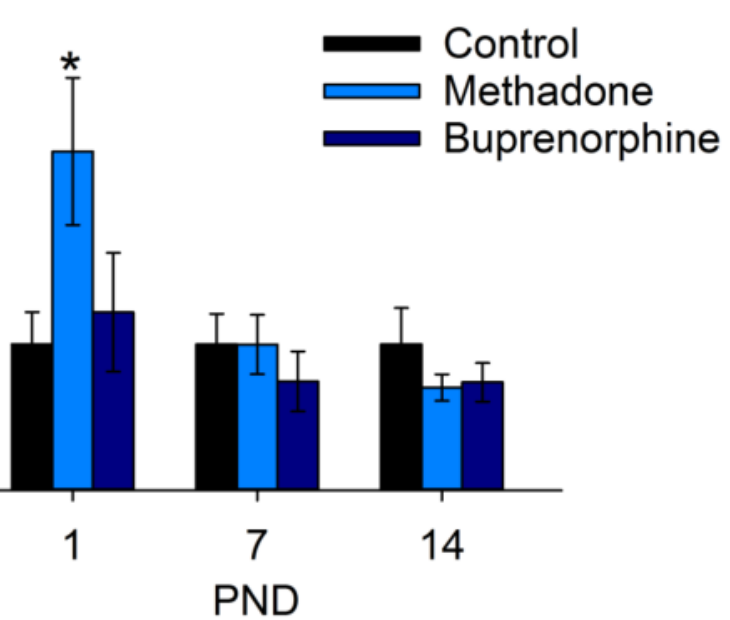

Figure 2. a) USV and b) blood concentrations of the stress hormone corticosterone (normalized to control) the first two weeks after birth in offspring prenatally exposed to methadone or buprenorphine. Results are shown as mean \pm SEM, $\mathrm{n}=8-10, * \mathrm{p}<0.05$.

Table 1. Neonatal outcomes on PND 1. Results are shown as mean \pm SEM.

\begin{tabular}{|lccc|}
\hline & Control & Methadone & Buprenorphine \\
\hline Body weight $(\mathrm{g})$ & $7.06 \pm 0.33$ & $6.54 \pm 0.28$ & $7.25 \pm 0.22$ \\
\hline Body length $(\mathrm{cm})$ & $5.26 \pm 0.13$ & $4.87 \pm 0.13^{*} \#$ & $5.31 \pm 0.10$ \\
\hline Brain weight $(\mathrm{g})$ & $0.26 \pm 0.01$ & $0.25 \pm 0.01$ & $0.26 \pm 0.02$ \\
\hline $\mathrm{n}=10,{ }^{*} \mathrm{p}<0.05$ (compared to control) ${ }^{\#} \mathrm{p}<0.05$ (compared to buprenorphine)
\end{tabular}

- The maternal blood concentrations of methadone and buprenorphine were stable throughout pregnancy, with concentrations of $198-238$ and $5.2-5.6 \mathrm{pmol} / \mathrm{mL}$, respectively. In comparison, clinical plasma concentrations of $500-600 \mathrm{pmol} / \mathrm{mL}$ methadone and $1-2 \mathrm{pmol} / \mathrm{mL}$ buprenorphine have been reported $[4,5]$.

- On PND 1, the administered opioid was present in both blood and brain from the offspring, with 8-9 times higher concentrations in brain tissue.

- On PND 3, pups prenatally exposed to methadone displayed increased USV. On PND 7, both methadone and buprenorphine exposed pups showed a tendency of a higher number of calls. Methadone exposed pups also displayed elevated levels of the stress hormone corticosterone on PND 1.

- On PND 1, offspring prenatally exposed to methadone displayed reduction in body length, and a tendency to weigh less compared to control and buprenorphine exposed offspring.

\section{CONCLUSION}

In this study, we present an exposure regimen to methadone or buprenorphine in rats giving stable and clinical relevant blood concentrations during pregnancy. Offspring prenatally exposed to methadone, but not buprenorphine, showed significant symptoms of abstinences. Methadone exposed offspring also demonstrated reduced birth length and a tendency of lower birth weight compared to controls and buprenorphine exposed offspring. These findings are similar to human observations [6]. Taken together, the presented exposure protocol provides a clinical relevant animal model to study outcomes in children born by mothers in OMT. 